\title{
SOLUBILITY AND THERMODYNAMIC MODELING OF QUETIAPINE FUMARATE IN SELF NANOEMULSIFYING DRUG DELIVERY SYSTEM (SNEDDS)
}

\author{
S. G. TALELE* , D. V. DERLE \\ Department: Pharmaceutics, N. D. M. V. P. College of Pharmacy \\ Email: swatitalele77@gmail.com \\ Received: 11 Mar 2018, Revised and Accepted: 06 Jun 2018
}

\section{ABSTRACT}

Objective: The present study gives emphasis on the development of self-nanoemulsifying drug delivery system (SNEDDS) of poorly water-soluble drug using Apelblat model.

Methods: For the development of self-nanoemulsifying drug delivery system (SNEDDS) solubility in surfactant, co-surfactant and in oil phase are considered as an important key to avoid phase separation and precipitation after dilution. The solubility of quetiapine fumarate was determined by the isothermal mechanical shaking method for its individual components in the temperature range from 305.15 to $330.15 \mathrm{~K}$ was measured. The experimental mole fraction solubility of quetiapine was good correlated with calculated data by using modified Apelblat model. Prepared SNEDDS were evaluated in centrifugation, freeze-thaw cycle study, self-nanoemulsification efficiency test. Physicochemical properties of prepared SNEDDS including particle size, zeta potential, viscosity and refractive index were carried out.

Results: The equilibrium saturated and mole fraction solubility of Quetiapine fumarate was found to be high in tween80 than SNEDDS, Labrafac lipophile WL 1349 and capryol 90. Quetiapine fumarate equilibrium saturated solubility, as well as mole fraction solubility, was found to be increased with increase in temperature in SNEDDS as well as in its individual components Prepared SNEDDS was found to be highly stable at centrifugation, heating and cooling cycles and freeze-thaw cycles and shows no sign of precipitation after dilution in water. All physicochemical parameters were observed within specification including droplet size observed as $26.37 \mathrm{~nm}$, polydispersity index 0.0970 , zeta potential-14.69 and the refractive index was observed as 1.458 which was nearer to the refractive index of water indicating the isotropic behavior of prepared SNEDDS.

Conclusion: The solubility study could be an effective approach for the development of thermodynamically stable SNEDDS formulation of poorly soluble drugs using Apelblat model.

Keywords: Quetiapine Fumarate, SNEDDS, Thermodynamic Modeling, Solubility Studies

(C) 2018 The Authors. Published by Innovare Academic Sciences Pvt Ltd. This is an open access article under the CC BY license (http://creativecommons.org/licenses/by/4.0/) DOI: http://dx.doi.org/10.22159/ijap.2018v10i4.25862

\section{INTRODUCTION}

Quetiapine fumarate is a psychotropic agent belonging to a chemical class of dibenzothiazepine derivatives. The chemical designation is 2-[2(4-dibenzo $[b, f][1,4]$ thiazepin-11-yl-1-piperazinyl) ethoxy]-ethanol fumarate (2:1) (salt). Quetiapine fumarate has an empirical formula C42H50N6O4S2 - C4H4O4 and molecular weight of 883.09. The biological life and bioavailability of QF is $6 \mathrm{~h}$ and 9\%, respectively (1-6). Quetiapine Fumarate is a BCS class II drug. Quetiapine fumarate is a weak acid with dissociation constant (pKa) 3.3 and 6.8 with moderate $\mathrm{pH}$-dependent solubility, $94.3 \mathrm{mg} / \mathrm{ml}$ to $2.37 \mathrm{mg} / \mathrm{ml}$ at $\mathrm{pH}$ values from 1 to 9 reported. The main site for absorption for quetiapine fumarate is stomach as it exhibits very high solubility in gastric $\mathrm{pH}$ which falls drastically in intestinal $\mathrm{pH}$. Thus, it would be more beneficial to retain quetiapine fumarate in the stomach (pH 1-3) for the prolonged duration to achieve maximum absorption and bioavailability [1-4].

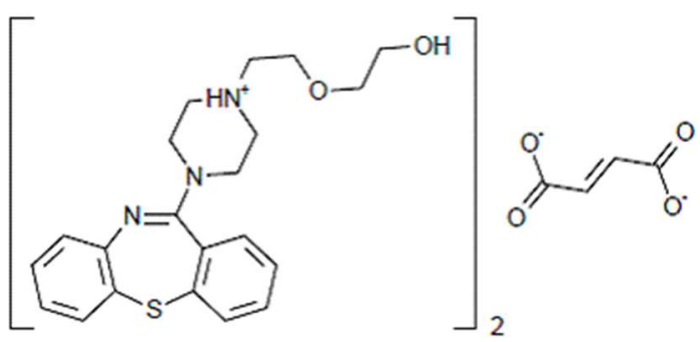

Fig. 1: Chemical structure of quetiapine fumarate

The self-nanoemulsifying drug delivery system (SNEDDS), which involves an isotropic Mixture of drug, surfactants and oil, is emerging as a means of augmenting the oral Bioavailability of hydrophobic drugs. This system produces (nano) emulsions after contact with aqueous medium with the gastrointestinal fluid $[5,6]$. These emulsion droplets are advantageous for drug absorption due to their large interfacial surface area, resulting in improved and reproducible bioavailability of poorly water-soluble drugs. SNEDDS is known to enhance the solubility of poorly soluble drugs, determination of drug solubility in surfactant, co-surfactant and in its oil phase and in SNEDDS is very essential The present paper focuses on the application of Thermodynamic based mathematical models for prediction of solubility of quetiapine fumarate in SNEDDS [7]. Thus the mole fraction solubility of quetiapine fumarate in prepared SNEDDS and its surfactant, cosurfactant and in oil phase components was predicted using empirical model equations and modified Apelblat model at the temperature range of 305.15 to $330.15 \mathrm{~K}$. This paper could illustrate the importance of mathematical modeling of solubility of poorly soluble drugs in SNEDDS in order to enhance solubility and oral bioavailability. The aim of this study was to provide a useful tool for mathematical modeling of poorly soluble drug in SNEDDS in order to enhance solubility, stability, dissolution and oral bioavailability.

\section{MATERIALS AND METHODS}

\section{Materials}

Quetiapine fumarate was received as gift samples from Wanbury Pharmaceuticals Ltd. (Mumbai, India). Caprylic/Capric Triglyceride, (Labrafac liphophile WL) Propylene glycol monocaprylate, (capryol 90)) was received as gift samples from Gattefosse (Mumbai, India). Tween 80 was purchased from Sigma Aldrich. All other chemicals and reagents were of highly purified grade and were used without further purification. All other chemicals used in the present study were of analytical reagent (AR grade). Their general properties are given in table 1 . 


\section{Methods}

\section{Determination of quetiapine fumarate solubility in SMEDDS and} its individual components

The saturated solubility of quetiapine fumarate in Labrafaclipophile, tween 80, capryol 90 and prepared SMEDDS were determined by using mechanical shaking method $[8,9]$ at atmospheric pressure and a temperature range from $305.15 \mathrm{~K}-330.15 \mathrm{~K}$. An excess amount of quetiapine fumarate was added in SNEDDS and its individual components in stoppered glass vials in triplicates. Each solid-liquid mixture vortexes properly to perform experiments. Each sample was then kept in a mechanical shaker at 100 RPM for $24 \mathrm{~h}$ to reach equilibrium. Each experiment was repeated at a temperature range from $305.15 \mathrm{~K}-330.15 \mathrm{~K}$. After $24 \mathrm{~h}$, all samples were taken out and allowed to settle drug (solute) particles for $2 \mathrm{~h}$ at the bottom of the glass vials. All the samples were subjected to centrifugation at 5000rpm for $15 \mathrm{~min}$, supernatant from each sample was taken and diluted with methanol and subjected to analysis of quetiapine fumarate using UV-Visible spectrophotometer at $246.5 \mathrm{~nm}$. From quetiapine fumarate content in each sample, equilibrium saturated solubility $(\mathrm{mg} / \mathrm{g}$ ) was determined. However, the experimental mole fraction solubility $\left(\mathrm{x}_{\mathrm{e}}\right)$ of quetiapine fumarate was calculated using equation 1 :

$$
\mathrm{Xe}=\frac{\mathrm{m} 1 / \mathrm{M} 1}{\mathrm{~m} 1 / \mathrm{M} 1+\mathrm{m} 2 / \mathrm{M} 2+\mathrm{m} 3 / \mathrm{M} 3+\mathrm{m} 4 / \mathrm{M} 4}
$$

Where $\mathrm{m} 1$ is the mass of quetiapine fumarate (solute) and $\mathrm{m} 2, \mathrm{~m} 3$ and $\mathrm{m} 4$ are the mass of solvents (Labrafaclipophile, tween 80 and capryol 90, respectively). M1 represents the molecular mass of quetiapine fumarate and M2, M3 and M4 represent the molecular masses of Labrafaclipophile, tween 80 and capryol 90, respectively.

Table 1: General properties of quetapine fumarate, oil, surfactant and co-surfactant

\begin{tabular}{lllll}
\hline Materials & Molecular formula & MW (g/Mol) & D (g/ml) & Chemical name \\
\hline Quetapine fumarate & $\mathrm{C}_{42} \mathrm{H}_{50} \mathrm{~N}_{6} \mathrm{O}_{4} \mathrm{~S}_{2} \bullet$ & 883.11 & - & $2-[2-(4-$ dibenzo [b,f] [1,4]thiazepin-11-yl-piperazinyl)ethoxy]-ethanol \\
& $\mathrm{C}_{4} \mathrm{H}_{4} \mathrm{O}_{4}$ & & & \\
Labrafac lipophile & $\mathrm{C} 16 \mathrm{H} 2605$ & 298.37 & $0.93-0.96$ & Caprylic/Capric Triglyceride \\
Tween 80 & $\mathrm{C} 64 \mathrm{H} 124026$ & 1310 & 1.075 & Polyoxyethylene (20) sorbitanmonooleate \\
Capryol 90 & $\mathrm{C} 11 \mathrm{H} 2203$ & 202.29 & $0.93-0.95$ & Propylene glycol monocaprylate \\
\hline
\end{tabular}

\section{Preparation of SNEDDS}

Various SNEDDS formulations of Quetiapine Fumarate were prepared by spontaneous emulsification technique via the construction of pseudoternary phase diagrams as reported previously 6 . Equilibrated saturated solubility of quetapine fumarate as shown in table 4 was carried out using mechanical shaking method and optimum composition of SNEDDS as shown in table 2 was formulated [6]
An optimum formulation of SNEDDS for solubility modeling was obtained by taking the required amount of Labrafil-Liphophile in a $5 \mathrm{~g}$ capacity vial. The required amount of Tween- 80 and Capryol 90 was added to oil phase and vortexed for $5 \mathrm{~min}$. Finally, required amount of distilled water (aqueous phase) was added to a mixture of oil phase and surfactant phase in dropwise manner till clear and transparent blank SNEDDS (without drug) obtained as shown in table 2.

Table 2: Composition of SNEDDS

\begin{tabular}{lll}
\hline S. No. & Formulation ingredients & Composition (\%w/w) \\
\hline 1 & Labrafac lipophile WL 1349 & 07.46 \\
2 & Tween 80 & 27.86 \\
3 & Capryol 90 & 14.92 \\
4 & Distilled water & 49.75 \\
\hline
\end{tabular}

\section{RESULTS AND DISCUSSION}

\section{Thermodynamic study and evaluation test}

Various SMEDDS formulations of quetiapine fumarate were developed and subjected to thermodynamic stability test to observe any phase separation, coalescence, creaming or conversion into the biphasic milky emulsion. Optimizes SNEDDS was selected for this study and was found to be highly stable at centrifugation, heating and cooling cycles and freeze-thaw cycles. Self-nano-emulsification efficiency test was carried out to evaluate signs of any precipitation or phase separation upon dilution with diluent. Distilled water was used as a diluent in this study. Prepared SNEDDS pass this test which indicated its suitability for SNEDDS.

\section{Physicochemical characterization of prepared SNEDDS}

The results of physicochemical characterization are listed in table 3 . The mean particle size of prepared SNEDDS was observed as $26.37 \mathrm{~nm}$ with polydispersity index (PI) 0.0970 . SNEDDS droplet size is a decisive point in the performance of self-emulsified formulations as it determines the rate and extent of drug release and absorption. Lowest value confirms its greatest absorption [6]. Polydispersability index below 0.3 indicates good uniformity in the globule size distribution after dilution with water. AS SNEDDS with increased globule size causes agglomeration of globules and suffers with the instability of the system [21]. The viscosity of SMEDDS was observed to be $0.890 \mathrm{cp}$. Indicating short time of emulsification compared to those formulations with higher viscosity. Zeta potential of SNEDDS was found to be$14.69 \mathrm{mv}$ which indicated the stable formation of SMEDDS. The negative value of zeta potential may be due to the presence of free fatty acids. A Negative value to the zeta potential of the optimized formulations indicated that the formulations were negatively charged, and sufficient repulsion among emulsion droplets existed to form an un-coagulated system and therefore, gives an indication of a stable system The Refractive Index (RI) of SNEDDS was observed at 1.458 which was very nearer to RI of water $(1.33)$. $[6,21]$ This indicated an isotropic behavior and o/w nature of prepared SNEDDS. Overall, all physicochemical parameters were found to suitable for SNEDDS.

\section{Table 3: Characterization of SNEDDS}

\begin{tabular}{lll}
\hline S. No. & Characterization parameters & Parameter value \\
\hline 1 & Droplet size (nm) & $26.37 \pm 0.208$ \\
2 & PI & $0.0970 \pm 0.0057$ \\
3 & Zeta potential (mv) & $-14.68 \pm 0.0057$ \\
4 & Viscosity (cp) & $0.890 \pm 0.01$ \\
5 & RI & $1.455 \pm 0.005$ \\
\hline
\end{tabular}

The values are expressed as mean $\pm \mathrm{SD} ; \mathrm{n}=3$, polydispersibility index (PI), refractive index (RI), 


\section{Solubility data of quetiapine fumarate}

The saturated equilibrium solubility of Quetiapine fumarate in Labrafac lipophile WL 1349, Tween 80, Capryol 90 and prepared SNEDDS at a temperature from 305 to $330 \mathrm{~K}$ are listed in table 4
The saturated equilibrium solubility of Quetiapine fumarate was found to be increased exponentially with increase in temperature in all sample matrices investigated. The saturated equilibrium solubility was observed highest in Tween 80 followed by Capryol 90, Labrafac lipophile WL1349 and SNEDDS as shown in table 6.

Table 4: Equilibrium saturated solubility of quetapine fumarate in labrafac lipophile wl 1349, tween 80, capryol 90 and SNEDDS

\begin{tabular}{|c|c|c|}
\hline Sample & Temperature (K) & Solubility (mg/g)* \\
\hline \multirow[t]{6}{*}{ Labrafaclipophile WL 1349} & 305.15 & $40.012 \pm 0.01$ \\
\hline & 310.15 & $55.341 \pm 0.371$ \\
\hline & 315.15 & $65.444 \pm 0.387$ \\
\hline & 320.15 & $81.514 \pm 0.347$ \\
\hline & 325.15 & $90.243 \pm 0.120$ \\
\hline & 330.15 & $110.410 \pm 0.245$ \\
\hline \multirow[t]{6}{*}{ Tween 80} & 305.15 & 115.000 \\
\hline & 310.15 & $121.671 \pm 0.190$ \\
\hline & 315.15 & $130.046 \pm 0.080$ \\
\hline & 320.15 & $145.001 \pm 0.00$ \\
\hline & 325.15 & $170.140 \pm 0.080$ \\
\hline & 330.15 & 200.000 \\
\hline \multirow[t]{6}{*}{ Capryol 90} & 305.15 & 58.000 \\
\hline & 310.15 & 66.000 \\
\hline & 315.15 & $75.445 \pm 0.145$ \\
\hline & 320.15 & $82.261 \pm 0.487$ \\
\hline & 32515 & $99.998 \pm 0.001$ \\
\hline & 330.15 & $123.05 \pm 0.522$ \\
\hline \multirow[t]{6}{*}{ SNEDDS } & 305.15 & $30.716 \pm 0.160$ \\
\hline & 310.15 & $39.544 \pm 0.264$ \\
\hline & 315.15 & $48.816 \pm 0.104$ \\
\hline & 320.15 & $65.583 \pm 0.505$ \\
\hline & 325.15 & $82.593 \pm 0.531$ \\
\hline & 330.15 & $95.990 \pm 0.0577$ \\
\hline
\end{tabular}

The values are expressed as mean \pm SD; $n=3$, self-Nano emulsifying drug delivery system (SNEDDS).

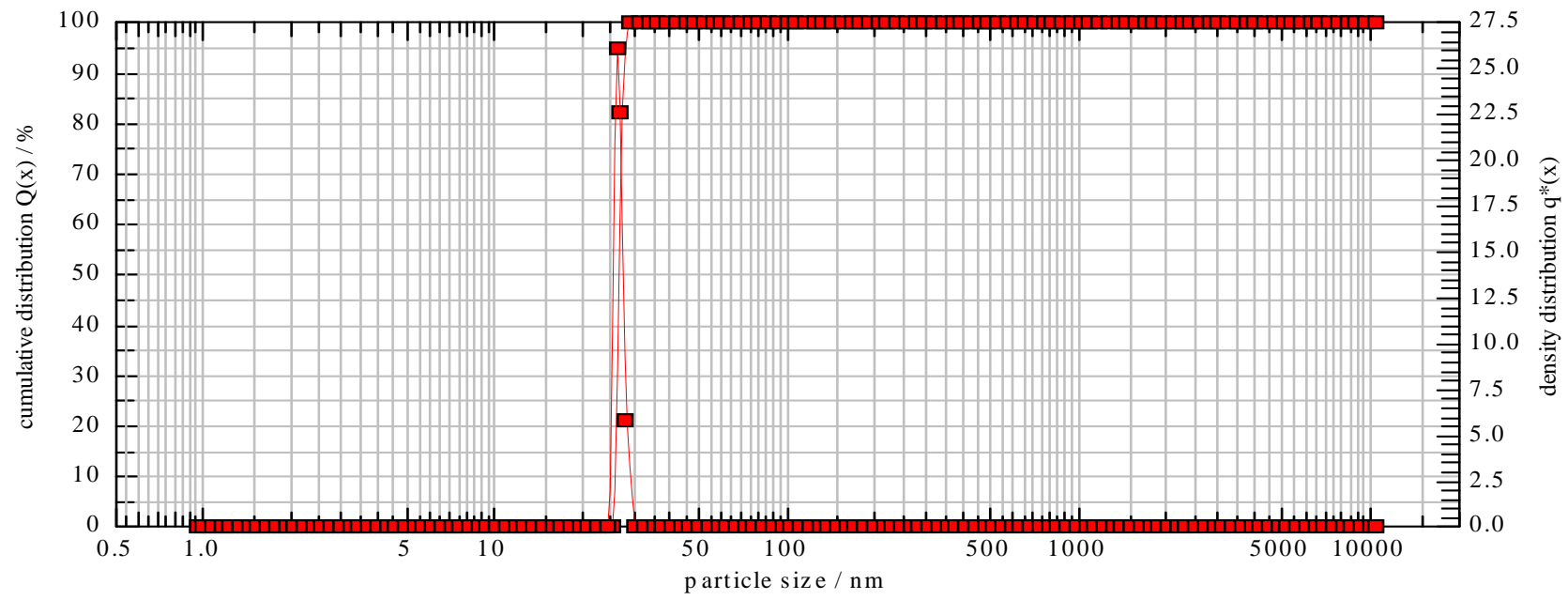

Fig. 2: Particle size of SNEDDS quetiapine fumarate 
Mobility Distribution

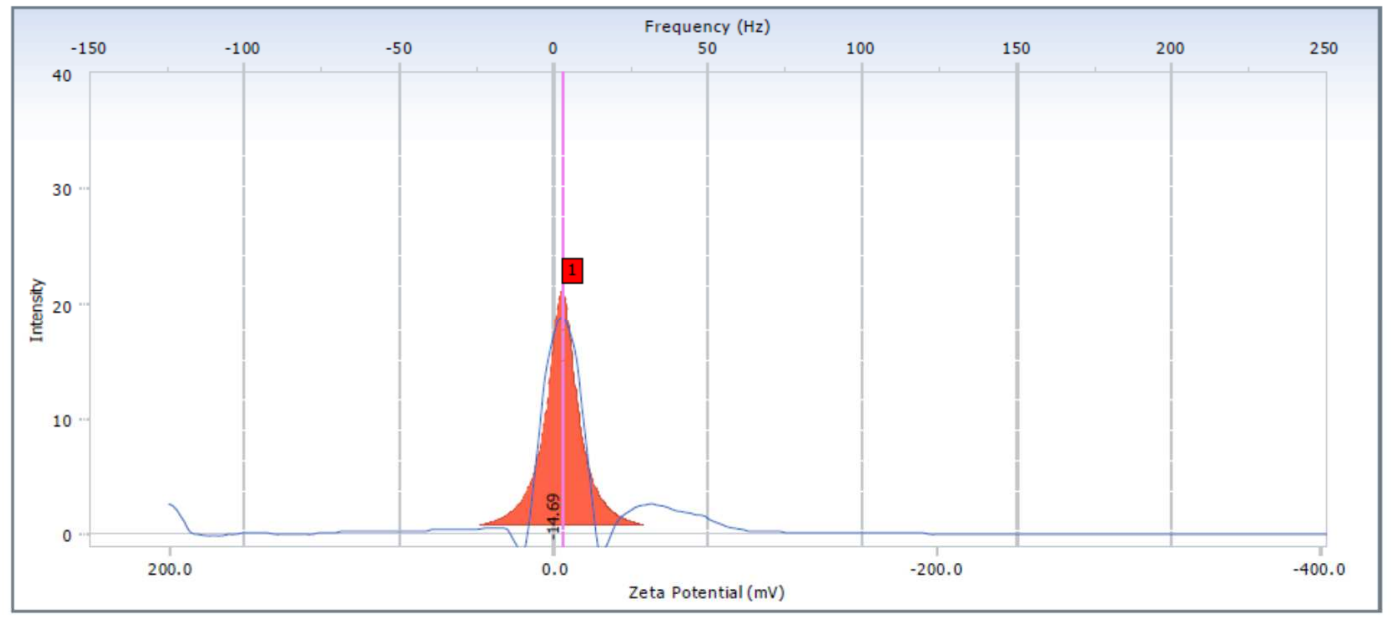

Measurement Results

Fig. 3: Zeta Potential of SNEDDS

Table 5: The modified apelblat model adjustable parameters for quetiapine fumarate in labrafac lipophile WL1349, tween-80, capryol 90 and prepared SNEDDS

\begin{tabular}{llll}
\hline Sample & Apelblat model & & \\
\cline { 2 - 4 } & A & B & C \\
\hline Labrafaclipophile WL1349 & 717.95 & -37108 & -104.991 \\
Tween 80 & -742.17 & 33192 & 110.381 \\
Capryol 90 & -691.95 & 29835 & 0.9919 \\
SNEDDS & 151.48 & -11405 & 0.9987 \\
\hline
\end{tabular}

Table 6: Equilibrium saturated solubility (S), mole fraction solubilities experimental (Xe) and calculated solubilities (xc) for quetiapine fumarate in labrafac lipophile labrafac lipophileWL1349, tween-80, tween 80, capryol 90 and prepared SNEDDS

\begin{tabular}{|c|c|c|c|c|c|c|}
\hline $\mathrm{T} / \mathrm{K}$ & $\mathrm{S}(\mathrm{mg} / \mathrm{g})^{*}$ & (xc) & ${ }^{103} \mathrm{XC}$ & $(\mathrm{Xe})^{*}$ & ${ }^{103} \mathrm{xe}^{*}$ & $\%$ AD \\
\hline \multicolumn{7}{|c|}{ Labrafac lipophile } \\
\hline \multicolumn{7}{|c|}{ WL 1349} \\
\hline 305.15 & $40.012 \pm 0.01$ & 0.01355 & 13.55 & $0.01326 \pm 0.00$ & $13.26 \pm 0.00$ & -2.27 \\
\hline 310.15 & $55.341 \pm 0.371$ & 0.01750 & 17.50 & $0.01825 \pm 0.001$ & $18.25 \pm 0.001$ & 4.19 \\
\hline 315.15 & $65.444 \pm 0.387$ & 0.02110 & 21.10 & $0.02151 \pm 0.101$ & $21.51 \pm 0.101$ & -1.40 \\
\hline 320.15 & $81.514 \pm 0.347$ & 0.026300 & 26.30 & $0.02665 \pm 0.002$ & $26.65 \pm 0.002$ & 1.42 \\
\hline 325.15 & $90.243 \pm 0.120$ & 0.030747 & 30.74 & $0.02942 \pm 0.001$ & $29.42 \pm 0.001$ & -4.62 \\
\hline 330.15 & $110.410 \pm 0.245$ & 0.034912 & 34.91 & $0.03576 \pm 0.020$ & $35.76 \pm 0.020$ & 2.44 \\
\hline \multicolumn{7}{|c|}{ Tween 80} \\
\hline 305.15 & 115.000 & 0.14544 & 145.44 & $0.1450 \pm 0.030$ & $145.0 \pm 0.030$ & -0.43 \\
\hline 310.15 & $121.671 \pm 0.190$ & 0.15131 & 151.31 & $0.1525 \pm 0.0102$ & $152.5 \pm 0.0102$ & 1.065 \\
\hline 315.15 & $130.046 \pm 0.080$ & 0.16174 & 161.74 & $0.1611 \pm 0.101$ & $161.1 \pm 0.101$ & -0.422 \\
\hline 320.15 & $145.001 \pm 0.00$ & 0.17731 & 177.31 & $0.1762 \pm 0.102$ & $176.2 \pm 0.102$ & -0.848 \\
\hline 325.15 & $170.140 \pm 0.080$ & 0.19904 & 199.04 & $0.2006 \pm 0.00$ & $200.6 \pm 0.00$ & 0.8110 \\
\hline 330.15 & 200.000 & 0.22845 & 228.45 & $0.2279 \pm 0.1012$ & $227.9 \pm 0.1012$ & -0.189 \\
\hline \multicolumn{7}{|c|}{ Capryol 90} \\
\hline 305.15 & 58.000 & 0.01321 & 13.21 & $0.013042 \pm 0.004$ & $13.04 \pm 0.004$ & 1.36 \\
\hline 310.15 & 66.000 & 0.01459 & 14.59 & $0.01482 \pm 0.102$ & $14.82 \pm 0.102$ & -1.615 \\
\hline 315.15 & $75.445 \pm 0.145$ & 0.01648 & 16.48 & $0.016894 \pm 0.001$ & $16.89 \pm 0.001$ & -2.54 \\
\hline 320.15 & $82.261 \pm 0.487$ & 0.01903 & 19.03 & $0.01845 \pm 0.203$ & $18.45 \pm 0.203$ & -0.40 \\
\hline 325.15 & $99.998 \pm 0.001$ & 0.02243 & 22.43 & $0.02227 \pm 0.402$ & $22.27 \pm 0.402$ & 0.77 \\
\hline 330.15 & $123.05 \pm 0.522$ & 0.02694 & 26.94 & $0.02726 \pm 0.502$ & $27.26 \pm 0.502$ & -1.20 \\
\hline \multicolumn{7}{|c|}{ SNEDDS } \\
\hline 305.15 & $30.716 \pm 0.160$ & 0.0119 & 11.9 & $0.0120 \pm 0.502$ & $12 \pm 0.502$ & 0.840 \\
\hline 310.15 & $39.544 \pm 0.264$ & 0.0154 & 15.4 & $0.0155 \pm 0.101$ & $15.5 \pm 0.101$ & 0.645 \\
\hline 315.15 & $48.816 \pm 0.104$ & 0.0197 & 19.7 & $0.0189 \pm 0.204$ & $18.9 \pm 0.204$ & -4.232 \\
\hline 320.15 & $65.583 \pm 0.505$ & 0.0249 & 24.9 & $0.0254 \pm 0.345$ & $25.4 \pm 0.345$ & 1.964 \\
\hline 325.15 & $82.593 \pm 0.531$ & 0.0311 & 31.1 & $0.0321 \pm 0.405$ & $32.1 \pm 0.405$ & 3.11 \\
\hline 330.15 & $95.990 \pm 0.0577$ & 0.0383 & 38.3 & $0.0375 \pm 0.407$ & $37.5 \pm 0.407$ & -2.133 \\
\hline
\end{tabular}

*The values are expressed as mean $\pm S D ; n=3$, calculated $(\mathrm{xc})$, experimental (xe), (\%AD). The percentage of absolute relative deviation. 


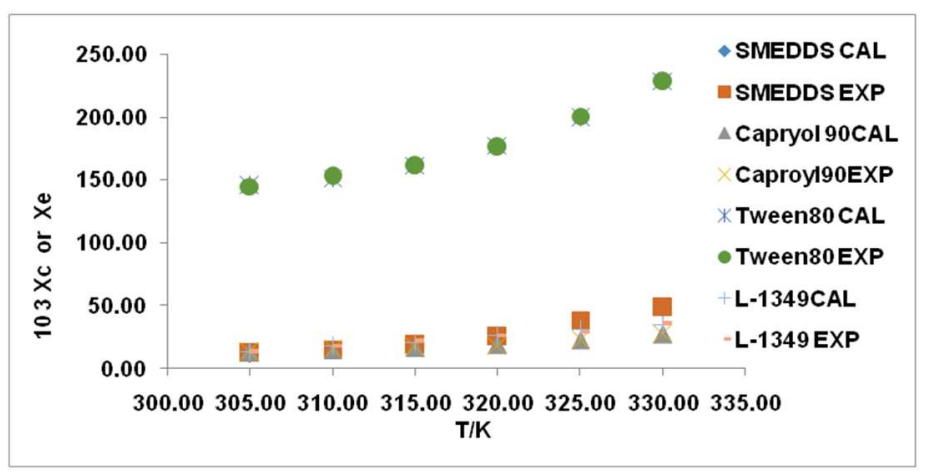

Fig. 4: Experimental and calculated mole fraction solubilities of quetiapine fumarate in tween 80, capryol 90, labrafacliphophile WL 1349 and self-nanoemulsifying drug delivery system (SNEDDS) at temperatures ranging from 300 to $335 \mathrm{~K}$

\section{Thermodynamic modeling of solubility of quetiapine fumarate}

Numerous models including NRTL, UNIQUAC, UNIFAC, COSMOPACE, GEQUAC have been used to correlate experimental mole fraction solubility with theoretical mole fraction solubility [10-20]. As the modified Apelblat model is more accurate and reliable model it was selected for correlation of experimental and calculated data. According to the modified Apelblat model, the temperaturedependent mole fraction solubilities of quetiapine fumarate at different temperatures can be calculated by the following equation:

In $\mathrm{Xe}=\mathrm{A}+\mathrm{B} / \mathrm{T}+\mathrm{CIn}(\mathrm{T})$

Where

$\mathrm{Xe}$ is the experimental mole fraction solubility of quetiapine fumarate

$\mathrm{T}$ is the absolute temperature $(\mathrm{k})$

$\mathrm{A}, \mathrm{B}$ and $\mathrm{C}$ are adjustable parameters.

The percentage of absolute relative deviation (\%AD) of Quetiapine fumarate was determined by using following equation (2)

\section{$\% \mathrm{AD}=\mathrm{Xe}-\mathrm{Xc} / \mathrm{Xe}$ x 100}

$\mathrm{Xe}$ and $\mathrm{Xc}$ indicates experimental and calculated mole fraction solubilites of quetiapine fumarate respectively. Experimental mole fraction solubilities (Xe) and Apelblat model correlation of Quetiapine fumarate in Capryol 90 and in Labrafil Liphofile WL 1349, Tween-80, and SNEDDS. are shown in fig. 4. The correlation coefficient value for quetiapine fumarate (R2) was obtained as $0.9920,0.9987,0.9940,0.9961$ for Labrafaclipophile WL 1349, Tween 80 , Capryol 90 and for SNEDDS as shown in table 6.

Thus solubility data were interrelated by experimental values and calculated values from the modified Apelblat equation and shows appropriate to each other.

\section{CONCLUSION}

In this thermodynamic modelling approach, the equilibrium saturated solubility, as well as the mole fraction solubility of quetiapine fumarate in prepared SNEDDS and its individual components in the temperature range from 305.15 to $330.15 \mathrm{~K}$, were measured. The solubility of quetiapine fumarate in surfactants tween 80 was found to be significantly higher than capryol 90, SNEDDS and labrafac liphophile WL1349. The solubility data of each experimental sample matrix were well correlated by the modified Apelblat model. The values of correlation coefficients (R2) in the range of 0.9960-0.9990 showed that the modified Apelblat model provided a good fitting of experimental solubility data of quetiapine fumarate. These preliminary studies on solubility could be a useful tool for the development of an efficient and thermodynamically stable SNEDDS formulation of various poorly soluble drugs to enhance their stability, solubility, dissolution and oral bioavailability.

\section{ACKNOWLEDGEMENT}

The authors are grateful to Dr. Alexander Apelblat Professor Emeritus Ben Gurion University of the Negev Chemical Engineering
Department for their greater contribution in the calculation. The authors are also thankful to Gattefosse (Mumbai) for providing the gift samples of Labrafac Liphophile 1349, Tween 80 and Transcutol.

\section{AUTHORS CONTRIBUTIONS}

All the author have contributed equally

\section{CONFLICT OF INTERESTS}

Declared none

\section{REFERENCES}

1. Brayfield A, Sweetman SC. Martindale, the complete drug reference. Pharmaceutical Press: London; 2007.

2. Andrew JC, Jeffrey MG, John AT. Dosing and switching strategies for quetiapine fumarate. Clin Ther 2002;24:209-22.

3. Talele S, Gudsoorkar VR. Novel approaches for solidification of SMEDDS. Int J Pharm Biosci 2016;15:90-101.

4. Ihor WR, Lisa AA. Overview of the efficacy of seroquel (quetiapine). Schi Res 1997;24:199.

5. King DJ, Link C. Seroquel (ICI 204636) an atypical antipsychotic result from phase III. E Neurol 1996;6:202.

6. SG Talele, DV Derle. Response surface methodology as a tool for optimization of self-nanoemulsified drug delivery system of quetiapine fumarate. Asian J Pharm 2018;11:319-30.

7. Bouillot B, Teychene S, Biscans B. An evaluation of thermodynamic models for the prediction of drug and drug-like molecule solubility in organic solvents. Fluid Phase Equilib 2011:309:36-52.

8. Jouyban A, Soltanpour S, Soltani S. Solubility prediction of drugs in water-cosolvent mixtures using Abraham solvation parameters. J Pharm Pharm Sci 2007;10:263-77.

9. Baka E, Comer JEA, Takac Novak K. Study of equilibrium solubility measurement by saturation shake-flask method using hydrochlorothiazide as a model compound. J Pharm Biomed Anal 2008;46:335-41.

10. Jimenez JA, Martinez F. Thermodynamic study of the solubility of acetaminophen in propylene glycolpwater cosolvent mixtures. J Braz Chem Soc 2006;17:125-34.

11. Yang GD, Huang YR, Nan GJ. Solubility of daidzein in the binary system of ethanol and water. J Mol Liq 2013;180:160-3.

12. Wang $Q$, Chen Y, Deng L. Determination of the solubility parameter of ionic liquid 1-alkyl-3-methylimidazolium chloride by inverse gas chromatography. J Mol Liq 2013;180:135-8.

13. Soltanpour S, Jouyban A. Solubility of lamotrigine in binary and ternary mixtures of $\mathrm{N}$-methyl pyrrolidone and water with polyethylene glycols 200, 400, and 600 at $298.2 \mathrm{~K}$. J Mol Liq 2013;180:1-6.

14. Yang W, Chen Z, Jiang X. Solubility of succinic anhydride in different pure solvents and binary solvent mixtures with the temperature range from 278.15 to 333.15 K. J Mol Liq 2013;180:7-11.

15. Domanska U, Pobudkowska A, Pelczarska A, Gierycz P pKa and solubility of drugs in water, ethanol, and 1-octanol. J Phys Chem B 2009;113:8941-7.

16. Sunsandee N, Hronec M, Stolcova M. Thermodynamics of the solubility of 4-acetyl benzoic acid in different solvents from 303.15 to $373.15 \mathrm{~K}$. J Mol Liq 2013;180:252-9. 
17. Wang L, Lv TT. Determination and modeling of the solubility and prediction of the dissolution properties of 2,4dichlorophenoxyacetic acid in toluene, tetrachloromethane and the binary solvent mixtures of (cyclohexanepethyl acetate). J Mol Liq 2013;181:29-33.

18. Zhang CL, Zhao F, Wang Y. Thermodynamics of the solubility of ciprofloxacin in methanol, ethanol, 1-propanol, acetone, and chloroform from 293.15 to $333.15 \mathrm{~K}$. J Mol Liq 2010;156:191-3.

19. Zhang CL, Zhao F, Wang Y. Thermodynamics of the solubility of sulfamethazine in methanol, ethanol, 1-propanol, acetone, and chloroform from 293.15 to $333.15 \mathrm{~K}$. J Mol Liq 2011;159:170-2.
20. Zhang CL, Li BY, Wang Y. Solubilities of norfloxacin in methanol, ethanol, 1-propanol, acetone, and chloroform from 294.15 to 318.15 K. Canadian J Chem Eng 2010;88:636.

21. Dharmang Pandya, Bhavin Rana, Nilay Solanki. Oral bioavailability enhancement of bromocryptine mesylate by the selfmicroemulsifyingdrug delivery system. Int J Pharm Pharm Sci 2016;8:76-81.

22. Shweta Khade, Yogesh Pare. Formulation and evaluation of neusilin US2 adsorbed amorphous solid self-microemulsifyingdelivery system of atorvastatin calcium. Asian J Pharm Clin Res 2016;9:93-10. 